\begin{tabular}{|c|l|}
\hline Title & Nano-scratching in solution to the single crystal Ta (100) subjected to anodic oxidation \\
\hline Author(s) & Seo, Masahiro; Kawamata, Daisuke \\
\hline Citation & $\begin{array}{l}\text { Journal of Physics D A pplied Physics, 39/15), 3150-3156 } \\
\text { https://doi.org/10.1088/0022-3727/39/15/304 }\end{array}$ \\
\hline Issue Date & 2006-08-07 \\
\hline Doc URL & http://hdl.handle.net/2115/14655 \\
\hline Rights & Copyright $\odot 2006$ IOP Publishing Ltd. \\
\hline Type & article (author version) \\
\hline File Information & JPD-A P2006-39-15.pdf \\
\hline
\end{tabular}

Instructions for use 


\title{
Nano-scratching in solution to the single-crystal Ta (100)
}

\section{subjected to anodic oxidation}

\author{
Masahiro Seo and Daisuke Kawamata \\ Graduate School of Engineering, Hokkaido University \\ Kia-13 Jo, Nishi-8 Chome, Kita-ku, Sapporo 060-8628, Japan \\ E-mail: $\underline{\text { seo@elechem1-mc.eng.hokudai.ac.jp }}$
}

\begin{abstract}
A single crystal-tantalum (100) surface was anodically oxidized at $5.0 \mathrm{~V}$ (SHE) for $1 \mathrm{~h}$ in $\mathrm{pH} 8.4$ borate buffer solution. Nano-scratching in solution at a constant normal force of $300 \mu \mathrm{N}$ was performed to the tantalum (100) surface kept at 5.0 V (SHE) after anodic oxidation. Nano-scratching in air was also performed to the tantalum (100) surface after anodic oxidation. A stick-slip was always observed in lateral force vs. lateral displacement curves during nano-scratching in solution. The degree of stick-slip, however, decreased with increasing scratching rate. The average friction coefficient obtained with nano-scratching in solution was significantly larger than that obtained with nano-scratching in air and increased linearly with logarithm of time required for scratching. In contrast, the friction coefficient obtained with nano-scratching in air was independent of time required for scratching. The protrusion at the scratching end of the groove produced by nano-scratching in solution was observed from the AFM image, while no protrusion was observed for nano-scratching in air. It has been proposed that the protrusion consists of anodic oxide accumulated on the moving front of the indenter tip during nano-scratching in solution and contributes to the increase in lateral force, i.e., friction coefficient.
\end{abstract}

\section{Introduction}

Tribocorrosion [1-3] is directly associated with the mechanical and electrochemical properties of passive metal surfaces, i.e., the mechanical breakdown of passive film, anodic dissolution of the substrate metal and repassivation are repeated during tribocorrosion. It was difficult so far to evaluate the mechanical properties of passive metal surfaces because the passive films are very thin (several nano-meters) .

The recent development of nano-indentation or nano-scratching technique [4-6], however, has made feasible to evaluate the mechanical properties of thin surface films on solids such as hardness, elastic modulus or friction coefficient. The nano-indentation or nano-scratching to the passive metal surfaces were mostly performed in air [7-9] after electrochemical preparation of passive films in solution. In our previous studies [10-12], nano-scratching in solution was attempted 
to the single-crystal iron or polycrystalline titanium surfaces kept at a constant potential in the passive region in solution and suceeded in measuring the friction coefficients of the passive metal surfaces in solution. As a result, the friction coefficients of passive surfaces obtained by nano-scratching in solution were significantly larger than those by nano-scratching in air after passivation. Moreover, the difference between friction coefficients in solution and in air for the passive titanium surface [11] was significantly larger than that for the passive iron surface [10,12], which may result from the high corrosion resistance of titanium as compared to iron.

The mechano-electrochemical reaction such as mechanical breakdown of passive film, active dissolution of the substrate and repassivation takes place during nano-scratching in solution as well as triobocorrosion. Particularly, the repassivation at the moving front of indenter would be promoted in solution under a potential control to provide high resistance against the lateral movement of indenter. The large friction coefficients of the passive surface in solution as compared to those in air were ascribed to the high repassivation rate at the moving front of indenter during nano-scratching in solution [10-12]. It is expected from the above mechano-electrochemical viewpoint that the passive metal surface with the higher repassivation rate has the larger friction coefficient in solution. The corrosion resistance of metal increases in the order of $\mathrm{Ta}>\mathrm{Ti}>\mathrm{Fe}$ and it depends on the repassivation rate. The difference between friction coefficients in solution and in air for the passive tantalum would be larger than that for the passive titanium.

In this paper, nano-scratching in solution was performed to a single-crystal tantalum (100) surface subjected to anodic oxidation to compare its friction coefficient with those of iron and titanium obtained previously and to make more clearly the effect of repassivation rate on friction coefficient.

\section{Experimental}

\subsection{Surface preparation}

A single-crystal tantalum (100) disk-plate with a diamter of $10 \mathrm{~mm}$ and a thickness of $1 \mathrm{~mm}$ was used for experiments. The tantalum specimen was mechanically polished with a diamond paste and finally electropolished with a constant current density of $78.4 \mathrm{~mA} \mathrm{~cm}^{-2}$ for $5 \mathrm{~min}$ in a mixture of $\mathrm{HF}$ $+\mathrm{H}_{2} \mathrm{SO}_{4}(1: 10)$ at $40{ }^{\circ} \mathrm{C}$ to remove a worked layer. The electrolyte solution employed for anodic oxidation was a $\mathrm{pH} 8.4$ borate buffer solution.

A small electrochemical cell made from Diflon was specially designed for nano-scratching in solution. The structure of the small electrochemical cell was described elsewhere $[10,13]$. Platinum ring and wire were used as counter and reference electrodes, respectively. The electrode potential was converted to the standard hydrogen electrode scale (SHE). The tantalum specimen was anodically oxidized at a constant current density of $50 \mu \mathrm{A} \mathrm{cm}^{-2}$ up to $5.0 \mathrm{~V}$ (SHE) in $\mathrm{pH} 8.4$ borate buffer solution and then kept at $5.0 \mathrm{~V}$ (SHE) for $1 \mathrm{~h}$. 


\subsection{Nano-scratching in solution}

The transducer (Hysitron Co. Ltd., Triboscope) for nano-scratching was attached to AFM (Digital Instruments, Nanoscope IIIa) in which the electrochemical cell was set. Lateral force, i.e., friction force, $F_{\mathrm{L}}$, and normal displacement, $D_{\mathrm{N}}$, can be simultaneously measured versus scratching distance, i.e., lateral displacement, $D_{\mathrm{L}}$, under a constant normal force, $F_{\mathrm{N}}$, by using this transducer. The spring constant of the transducer was $206 \mathrm{~N} \mathrm{~m}^{-1}$ for the normal direction (z-axis) and $468 \mathrm{~N} \mathrm{~m}^{-1}$ for the lateral direction (x-axis), respectively.

The nano-scratching in solution to the passive tantalum surface kept at $5.0 \mathrm{~V}$ (SHE) was carried out at various scratching rates of $v=0.2$ to $1.0 \mu \mathrm{m} \mathrm{s}^{-1}$ up to a lateral distance of $2 \mu \mathrm{m}$ under a constant normal force of $F_{\mathrm{N}}=300 \mu \mathrm{N}$ by using a conical diamond indenter with a tip radius less than $1 \mu \mathrm{m}$ and with an included angle of $90^{\circ}$. The diamond tip was attached to a tungsten rod for use in liquid. After the nano-scratching, the morphology of the scratched area was observed with AFM by using the same diamond tip for the nano-scratching. The nano-scratching in air after anodic oxidation was also performed under the same conditions employed for nano-scratching in solution for comparison.

\section{Results and discussion}

3.1. Normal displacement, $D_{\mathrm{N}}$, and lateral force, $F_{\mathrm{L}}$, versus lateral displacement, $D_{\mathrm{L}}$, curves

Figure 1 shows the $D_{\mathrm{N}}$ and $F_{\mathrm{L}}$ vs. $D_{\mathrm{L}}$ curves obtained with nano-scratching in solution at scratching rates of (a) $v=0.4 \mu \mathrm{m} \mathrm{s}^{-1}$ and (b) $v=1.0 \mu \mathrm{m} \mathrm{s}^{-1}$ for the passive tantalum (100) surface subjected to anodic oxidation. The value of $D_{\mathrm{N}}$ on the ordinate was taken positive, referring the surface level before scratching to zero. The value of $F_{\mathrm{L}}$ increased accompanying a periodical fluctuation as $D_{\mathrm{L}}$ increased, and did not attain a steady state, i.e., a stick-slip was observed, which was significant at the lower scratching rate. Figure 2 shows the $D_{\mathrm{N}}$ and $F_{\mathrm{L}}$ vs. $D_{\mathrm{L}}$ curves obtained with nano-scratching in air at scratching rates of (a) $v=0.4 \mu \mathrm{m} \mathrm{s}^{-1}$ and (b) $v=1.0 \mu \mathrm{m} \mathrm{s}^{-1}$ after anodic oxidation. The value of $F_{\mathrm{L}}$ increased rapidly at the initial stage and then attained a steady state, i.e., no stick-slip was observed for nano-indentation in air. From the comparison between figures 1 and 2, it is noted that the average values of $F_{\mathrm{L}}$ and $D_{\mathrm{N}}$ for nano-scratching in solution are larger by factors of 8 and 1.5, respectively, than those for nano-scratching in air, which may result from a series of mechano-electrochemical reaction taking place during nano-scratching in solution.

It has been reported $[14,15]$ that a stick-slip does not occur at any scratching rates in the case where the spring constant, $k$, for $\mathrm{x}$-axis exceeds a critical value, and the scratching takes a steady state. On the other hand, in the case where $k$ is less than a critical value, the transition from steady-state to stick-slip takes place at the lower scratching rate $[14,15]$. Assuming the average lateral force, $F_{\mathrm{L}}=300 \mu \mathrm{N}$ during nano-scratching in solution (see figure 1 (a)), the transducer would be strained by $0.64 \mu \mathrm{m}$ to the lateral direction, since the spring constant, $k$, of the transducer is 468 $\mathrm{N} \mathrm{m}^{-1}$ for $\mathrm{x}$-axis. It seems that the magnitude of the strain is enough to induce a stick-slip. The 
thickness of passive film formed on tantalum (100) at 5.0 V (SHE) for $1 \mathrm{~h}$ is estimated to be about 13 $\mathrm{nm}$ from the linear relation between ellipsometrical thickness and film formation potential which was previously obtained for a polycrystalline tantalum [16]. The normal displacement, $D_{\mathrm{N}}$, during nano-scratching in solution or in air is larger by a factor of 4.6 or 3.1 than the film thickness as seen from the $D_{\mathrm{N}}$ vs. $D_{\mathrm{L}}$ curves in figures 1 and 2 . This means that not only the passive film but also the substrate tantalum is removed during nano-scratching.

\subsection{Difference in morpholgy of the scratched areas between nano-scratching in solution and air}

Figures 3 shows the AFM images of the scratched areas after (a) nano-scratching in solution and (b) nano-scratching in air at a scratching rate of $v=0.4 \mu \mathrm{m} \mathrm{s}^{-1}$. The grooves produced by nano-scratching can be observed from the AFM images. It is remarkable for nano-scratching in solution that a protrusion is present at the scratching end of the groove. The presence of protrusion can be clearly confirmed from the cross section of the groove in figure 3 (a). In contrast, for nano-scratching in air, no significant protrusion is present at the scratching end of the groove as seen from figure 3 (b). It is also seen from the cross section of the groove that the depth of the groove for nano-scratching in solution is larger than that for nano-scratching in air, which is consistent with the results of the $D_{\mathrm{N}}$ vs. $D_{\mathrm{L}}$ curves in figures 1 and 2 .

Figures 4 shows the AFM images of the scratched areas after (a) nano-scratching in solution and (b) nano-scratching in air at a scratching rate of $v=0.8 \mu \mathrm{m} \mathrm{s}^{-1}$. A protrusion can be observed at the scratching end of the groove for nano-scratching in solution. The amount of the protrusion, however, tends to decrease at the higher scratching rate as seen from the comparison between figures 3 (a) and 4 (a). On the other hand, no significant protrusion can be observed for nano-scratching in air even if the scratching rate is changed. The protrusion observed at the end of the groove for nano-scratching in solution should be directly associated with the large value of $F_{\mathrm{L}}$ accompanying a stick-slip. The protrusion may be formed by a series of mechano-electrochemical reaction taking place at the moving front of the indenter tip during nano-scratching in solution. The formation mechanism of the protrusion will be discussed later to explain the anomalous behavior of nano-scratching in solution.

\subsection{Average friction coefficient, $\mu_{\text {ave, }}$, as a function of scratching rate, $v$}

The degree of stick-slip observed for nano-scratching in solution was the more severe at the lower scratching rate. The friction coefficient, $\mu$, is defined with dividing $F_{\mathrm{L}}$ by $F_{\mathrm{N}}$ [17].

$\mu^{\prime}=\frac{F_{L}}{F_{N}}$

In our previous study on nano-scratching in solution to the passive iron surface $[10,12]$, a steady-state value of $F_{\mathrm{L}}$ under a constant value of $F_{\mathrm{N}}$ could be employed for evaluation of $\mu$ ' since no stick-slip was observed. In the case of nano-scratching in solution to the passive tantalum surface 
where a stick-slip takes place, an average friction coefficient, $\mu_{\text {ave }}$, defined as follows may be convenient to explain the anomalous behavior of nano-scratching in solution which may be associated with a series of mechano-electrochemical reaction at the moving front of the indenter tip.

$$
\mu_{\text {ave }}^{\prime}=\frac{W}{D_{L} F_{N}}
$$

$W=\int_{0}^{D_{L}} F_{L} d D_{L}$

where $W$ corresponds to the irreversible work done by the indenter tip during nano-scratching. The value of $W$ can be estimated by a graphic integration of the $F_{\mathrm{L}}$ vs. $D_{\mathrm{L}}$ curve from $D_{\mathrm{L}}=0$ to $2 \mu \mathrm{m}$ as shown schematically in figure 5. Figure 6 shows the average friction coefficient, $\mu_{\text {ave }}^{\prime}$, thus estimated as a function of scratching rate, $v$. The value of $\mu_{\text {ave }}$ obtained with nano-scratching in solution decreases with increasing $v$, while that obtained with nano-scratching in air is independent

of $v$. The time, $\tau$, required for scratching is given by $\tau=\frac{D_{L}}{v}$. The value of $\mu_{\text {ave }}$ is replotted versus $\operatorname{logarithm}$ of $\tau$ in figure 7. The linear relation between $\mu_{\text {ave }}^{\prime}$ and $\log \tau$ holds within experimetal errors for nano-scratching in solution, which is quite similar to the linear relation between $\mu^{\prime}$ and $\log v$ observed usually at the low scratching rate in the steady-state scratching regime[14, 15].

\subsection{Formation mechanism of the protrusion proposed to explain the anomalous behavior of nano-scratching in solution}

The large value of the average friction coefficient and its dependence of scratching rate for nano-scratctching in solution would result from the formation of protrusion on the moving front of the indenter tip. Both anodic oxide film with a thickness of $13 \mathrm{~nm}$ and substrate metal layer with a thickness of $50 \mathrm{~nm}$ are mechanically removed during nano-scratching in solution to produce a bare metallic groove which will be anodically oxidized again to form oxide film after the indenter tip passed. Moreover, the removed metallic tantalum scrap can be converted to anodic oxide due to high electric field since the potential of the specimen is kept at 5.0 V (SHE) in solution. Particularly, some fraction of the metallic scrap will easily stick and accumulate as anodic oxide on the moving front of the indenter tip. The electrochemical conversion of metallic tantalum to anodic oxide can be formulated as follows.

$2 \mathrm{Ta}+5 \mathrm{H}_{2} \mathrm{O} \rightarrow \mathrm{Ta}_{2} \mathrm{O}_{5}+10 \mathrm{H}^{+}+10 e^{-}$

Although the anodic current should flow to form anodic oxide during nano-scratching in solution, it was impossible to detect experimentally the anodic current which was very small and was masked with a noisy background current.

It is deduced from the above mechano-electrochemical viewpoint that the protrusion observed at the scratching end of the groove consists of tantalum anodic oxide. At present, however, it is not made clear the reason why tantalum or its anodic oxide sticks easily on the moving front of the indenter tip. The electrochemical conversion of metallic tantalum to anodic oxide leads to a 
volume expansion by a factor of 2.5 due to the difference in density between metallic tantalum and tantalum anodic oxide [18]. This large volume expansion may promote the adherence of anodic oxide on the moving front of the indenter. On the other hand, in the case of nano-scratching in air, the removed metallic scrap could not be easily converted to oxide since air-oxidation is a slow process which is only brought by oxygen in air. Even if a thin air-formed oxide sticks on the moving front of the indenter tip, the protrusion is negligibly small and could not be observed with AFM.

Air-formed film and anodic oxide film on tantalum have amorphous structures with fine crystallines of $\beta-\mathrm{Ta}_{2} \mathrm{O}_{5}$ [19]. Although the mechanical properties of the tantalum amorphous oxide are unknown, the recent nano-indentation test indicated that the hardness of tantalum anodic oxide was three times as much as that of the substrate metallic tantalum [20]. There is some correlation between hardness and yield strength for materials [21]. The yield strength of tantalum anodic oxide, therefore, may be larger than that of metallic tantalum. It is generally accepted that the lateral force, $F_{\mathrm{L}}$, consists of the adhesion term and the ploughing term [16]. It seems that the ploughing term predominates over the adhesion term under the present nano-scratching conditions since the surface layer with a thickness of $40-60 \mathrm{~nm}$ was really removed by the scratching, which corresponds to the abrasive wear regime $[22,23]$. The criterion representing the degree in wear is the specific wear rate or specific wear amount, $r_{\mathrm{w}}\left(\mathrm{mm}^{2} \mathrm{~N}^{-1}\right)$, [22] given by

$r_{w}=\frac{V}{F_{N} D_{L}}$

where $V$ is the wear volume. The wear volume, $V$, can be calculated from the bottom depth, $d$, of the groove with a triangular cross section by taking the geometry of the indenter tip into account.

$V=d^{2} D_{L}$

The bottom depth, $d$, of the groove can be estimated from the AFM image of the scratched area (see figures 3 and 4). The values of $r_{\mathrm{w}}$ thus obtained were $1.2 \times 10^{-5} \mathrm{~mm}^{2} \mathrm{~N}^{-1}$ for nano-scratching in solution and $5.3 \times 10^{-6} \mathrm{~mm}^{2} \mathrm{~N}^{-1}$ for nano-scratching in air, both of which were in the range of abrasive wear $\left(r_{\mathrm{w}}=10^{-5}-10^{-7} \mathrm{~mm}^{2} \mathrm{~N}^{-1}\right)$ [22].

In the case where the ploughing term is predominant, the accumulation of tantalum anodic oxide on the moving front of the indenter tip would give the large resistance against the lateral movement of the indenter and contribute to the increase in lateral force, i.e., friction coefficient. If the lateral force is proportional to the amount of anodic oxide accumulated on the moving front of the indenter tip, the linear relation between $\mu_{\text {ave }}$ and $\log \tau$ may be derived from the growth kinetics of anodic oxide obeying the high field model [3]. Assuming that some fraction of surface metallic tantalum removed by scratching sticks on the moving front of the indenter and grows as anodic oxide, we have recently calculated the field-asisted anodic oxide growth by taking in account the difference in initial integration time at each segment of the groove [3], and eventually indicated that the amount of anodic oxide accumulated on the moving front of the indenter was almost proportional to $\log \tau$ [24]. 
In our previous study [10,12], the large friction coefficient of the passive iron surface obtained with nano-scratching in solution as compared to nano-scratching in air was ascribed to the high repassivation rate at the moving front of the indenter. In our present study, however, the accumulation of anodic oxide in addition to the high repassivation rate has to be taken into account to explain the anomalous behavior of nano-scratching in solution, accompanying a stick-slip for the passive tantalum surface.

\section{Summary}

Nano-scratching in solution under a constant normal force, $F_{\mathrm{N}}=300 \mu \mathrm{N}$ was performed to the single-crystal tantalum (100) surface kept at 5.0 V (SHE) after anodic oxidation at $5.0 \mathrm{~V}$ (SHE) for 1 $\mathrm{h}$ in $\mathrm{pH} 8.4$ borate buffer solution. The results were summarized as follows.

1) A stick-slip was observed during nano-scratching in solution and was significant at the lower scratching rate, while no stick-slip was observed during nano-scratching in air after anodic oxidation.

2) The average friction coefficient, $\mu_{\text {ave }}$, could be obtained with a graphic integration of the lateral force, $F_{\mathrm{L}}$, vs. lateral displacement, $D_{\mathrm{L}}$, curve. The value of $\mu_{\text {ave }}^{\prime}$ for nano-scratching in solution was significantly larger than that for nano-scratching in air. Particularly, the value of $\mu_{\text {ave }}^{\text {a for }}$ nano-scratching in solution increased linearly with increasing logarithm of time, $\tau$, required for scratching, while that for nano-scratching in air was independent of $\tau$.

3) The protrusion at the scratching end of the groove for nano-scratching in solution was observed from the AFM image of the scratched area, and the amount of protrusion tended to decrease at the high scratching rate. In contrast, no protrusion at the scraching end of the groove for nano-scratching in air was obseved from the AFM image, irrespective of scratching rate.

4) It was assumed that the protrusion consists of tantalum anodic oxide accumulated on the moving front of indenter to explain the anomalous behavior of nano-scratching in solution and the large value of $\mu_{\text {ave }}^{\prime}$. The present nano-scratching conditions for the tantalum surface ranged in the abrasive wear. Some fraction of the metallic tantalum removed by nano-scratching in solution would stick easily on the moving front of the indenter tip and could be converted to anodic oxide due to high electric field.

5) In the abrasive wear regime where the ploughing term prodomunates over the adhesive term for lateral force, it has been proposed that the accumulation of tantalum anodic oxide on the moving front of the indenter tip gives the large resistance against the lateral movement of the indenter tip and contributes to the increase in lateral force, i.e., friction coefficient.

\section{References}

[1] Mischler S, Debaud S and Landolt D 1998 J. Electrochem. Soc. 145750

[2] Mischler S, Spiegel A and Landolt D 1999 Wear 225-229 1078 
[3] Jemmely P, Mischler S and Landolt D 2000 Wear 23763

[4] Oliver W C and Pharr G M 1992 J. Mater. Res. 71564

[5] Harvey S E, Huang H, Venkataraman S K and Gerberich W W 1993 J. Mater. Res. 81291

[6] Bhushan B 1995 Handbook of Micro/Nano Tribology, ed B Bhushan (New York: CRC) p 321

[7] Gerberich W W, Venkataraman S K, Hunag H, Harvey S E and Kohlstedt D L 1995 Acta mater. 431569

[8] Pang M, Eakins D E, Norton M G and Bahr D F 2001 Corrosion 57523

[9] Alcalá S, Mato S, Skeldon P, Thompson G E, Mann A B, Habazaki H and Shimizu K 2003 Surf. Coat. Technol. 173293

[10] Chiba M and Seo M 2003 J. Electrochem. Soc. 150 B525

[11] Seo M and Kurata Y 2003 Electrochim. Acta 493221

[12] Chiba M and Seo M 2004 Electrochim. Acta 50967

[13] Chiba M and Seo M 2002 Corros. Sci. 44379

[14] Heslot F, Baumberger T, Perrin B, Carili B and Caroli C 1994 Phys. Rev. E 494973

[15]Persson B N J 1998 Sliding Friction-Physical Principles and Applications (Berlin: Springer) p 368

[16] Seo M, Kawamata D and Chiba M 2006 Differences in mechanical properties of the passive metal surface obtained in solution and air Passivations of Metals and Semiconductor, and Properties of Thin Oxide Layers: Proc. Passivity-9 (Paris, France, 27 June-1 July 2005) ed P Marcus and V Maurice (Amsterdam: Elsevier) in print

[17] Moore D F 1975 Principles and Applications of Tribology (Oxford: Pergamon) p 33

[18] Weast R C 1980-1981 Handbook of Chemistry and Physics (Florida: CRC Press) p B-154

[19] Aleshina L A, Malinenko V P, Phouphanov A D and Jakovleva N M 1986 J. Non-Crys. Solids 87350

[20] Kawamata D and Seo M 2006 Mechanical properties of single-crystal tantalum (100) surface covered with anodic oxide film Passivations of Metals and Semiconductors, and Properties of Thin Oxide Layers: Proc. Passivity-9 (Paris, France, 27 June-1 July 2005) ed P Marcus and V Maurice (Amsterdam: Elsevier) in print

[21] Tabor D 1985 Microindentation Techniques in Materials Science and Engineering Microindentation Hardness Symposium and Workshop (Philadelphia, USA, 15-18 July 1984) ed P J Blau and B R Lawn (Philadelphia: ASTM and IMS) p 134

[22] Yamamoto Y and Kaneta M 1998 Tribology (Japanese) (Tokyo: Rikohgakusha) p 189

[23] Lim S C and Ashby M F 1987 Acta metall. 351

[24] Seo M and Kawamata D 2005 Nano-scratching in solution to the Ta (100) surface subjected to anodic oxidation Abs. Symp. 4-B Corrosion and Surface Treatments, 56 th Annual Meeting of the International Society of Electrochemistry (Busan, Korea 25-30 September 2005) p 189 


\section{Figure Captions}

Figure 1. Normal displacement, $D_{\mathrm{N}}$, and lateral force, $F_{\mathrm{L}}$, versus lateral displacement, $D_{\mathrm{L}}$, curves obtained with nano-scratching in solution at scratching rates of (a) $v=0.4 \mu \mathrm{m} \mathrm{s}^{-1}$ and (b) $v=1.0 \mu \mathrm{m}$ $\mathrm{s}^{-1}$ for the passive tantalum (100) surface subjected to anodic oxidation at $5.0 \mathrm{~V}$ (SHE) in pH 8.4 borate buffer solution.

Figure 2. Normal displacement, $D_{\mathrm{N}}$, and lateral force, $F_{\mathrm{L}}$, versus lateral displacement, $D_{\mathrm{L}}$, curves obtained with nano-scratching in air at scratching rates of (a) $v=0.4 \mu \mathrm{m} \mathrm{s}^{-1}$ and (b) $v=1.0 \mu \mathrm{m} \mathrm{s}^{-1}$ for the passive tantalum (100) surface subjected to anodic oxidation at $5.0 \mathrm{~V}$ (SHE) in pH 8.4 borate buffer solution.

Figure 3. AFM images of the scratched areas after (a) nano-scratching in solution and (b) nano-scratching in air at a scratching rate of (a) $v=0.4 \mu \mathrm{m} \mathrm{s}^{-1}$ for the passive tantalum (100) surface subjected to anodic oxidation at $5.0 \mathrm{~V}$ (SHE) in $\mathrm{pH} 8.4$ borate buffer solution. The lower figures represent the cross sections of the grooves in the scratching direction drawn with the dashed lines in the AFM images.

Figure 4. AFM images of the scratched areas after (a) nano-scratching in solution and (b) nano-scratching in air at a scratching rate of $v=0.8 \mu \mathrm{m} \mathrm{s}^{-1}$ for the passive tantalum (100) surface subjected to anodic oxidation at $5.0 \mathrm{~V}$ (SHE) in $\mathrm{pH} 8.4$ borate buffer solution. The lower figures represent the cross sections of the grooves in the scratching direction drawn with the dashed linse in the AFM images.

Figure 5. Irreversible work, $W$, done by the indeter tip during nano-scratching which is estimated by a graphic integration of the $F_{\mathrm{L}}$ vs. $D_{\mathrm{L}}$ curve from $D_{\mathrm{L}}=0$ to $2 \mu \mathrm{m}$.

Figure 6. Relation between average friction coefficient, $\mu_{\text {ave }}^{\prime}$, and scratching rate, $v$, obtained with nano-scratching in solution and in air.

Figure 7. Relation between average friction coefficient, $\mu_{\text {ave }}$, and logarithm of time, $\tau$, required for scratching. 

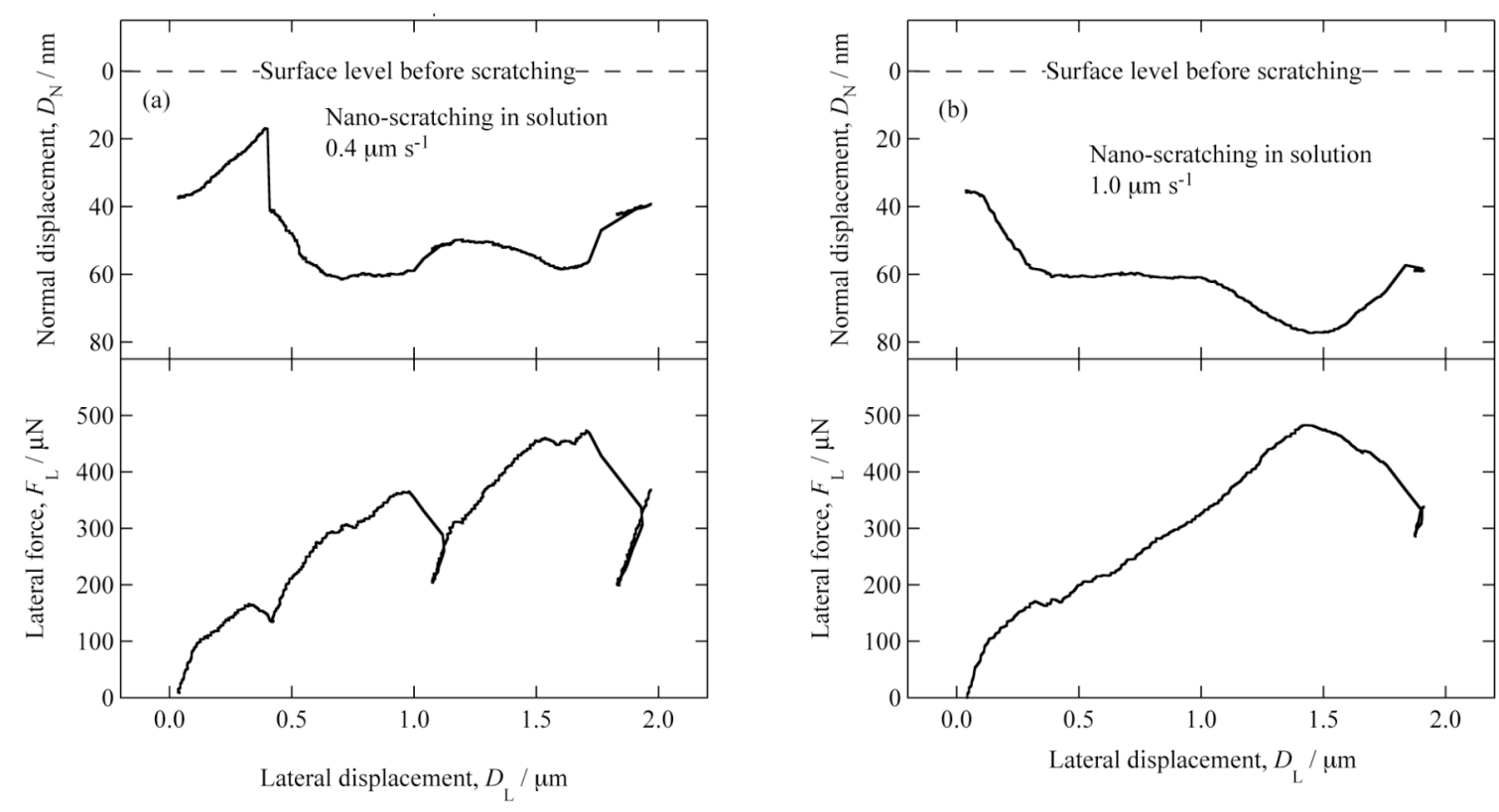

Figure 1. Normal displacement, $D_{\mathrm{N}}$, and lateral force, $F_{\mathrm{L}}$, versus lateral displacement, $D_{\mathrm{L}}$, curves obtained with nano-scratching in solution at scratching rates of (a) $v=0.4 \mu \mathrm{m} \mathrm{s}^{-1}$ and (b) $v=1.0 \mu \mathrm{m} \mathrm{s}^{-1}$ for the passive tantalum (100) surface subjected to anodic oxidation at $5.0 \mathrm{~V}$ (SHE) in $\mathrm{pH} 8.4$ borate buffer solution. 

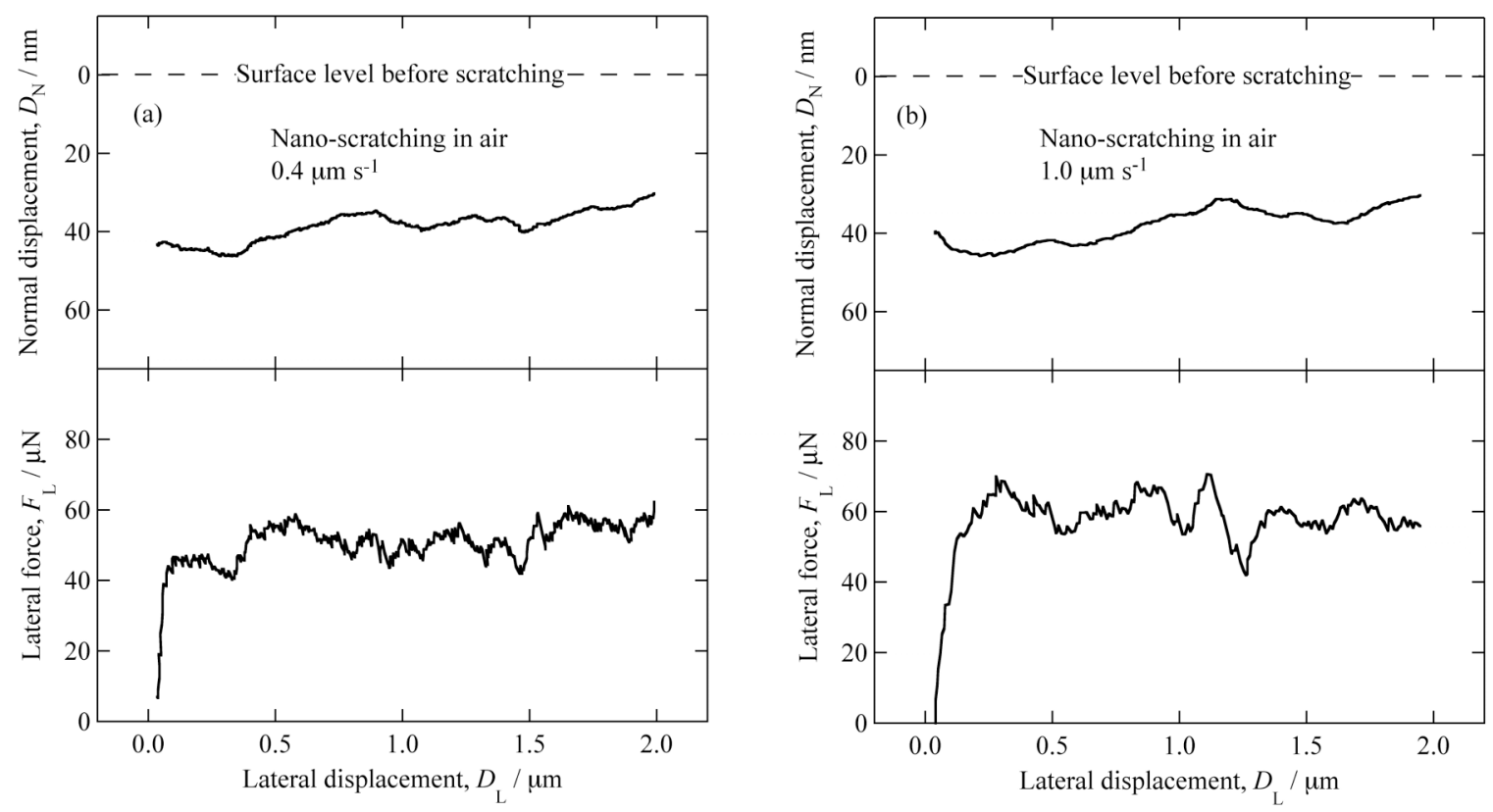

Figure 2. Normal displacement, $D_{\mathrm{N}}$, and lateral force, $F_{\mathrm{L}}$, versus lateral displacement, $D_{\mathrm{L}}$, curves obtained with nano-scratching in air at scratching rates of (a) $v=0.4 \mu \mathrm{m} \mathrm{s}^{-1}$ and (b) $v=1.0 \mu \mathrm{m} \mathrm{s}^{-1}$ for the passive tantalum (100) surface subjected to anodic oxidation at $5.0 \mathrm{~V}$ (SHE) in $\mathrm{pH} 8.4$ borate buffer solution. 
(a) Nano-scratching in solution
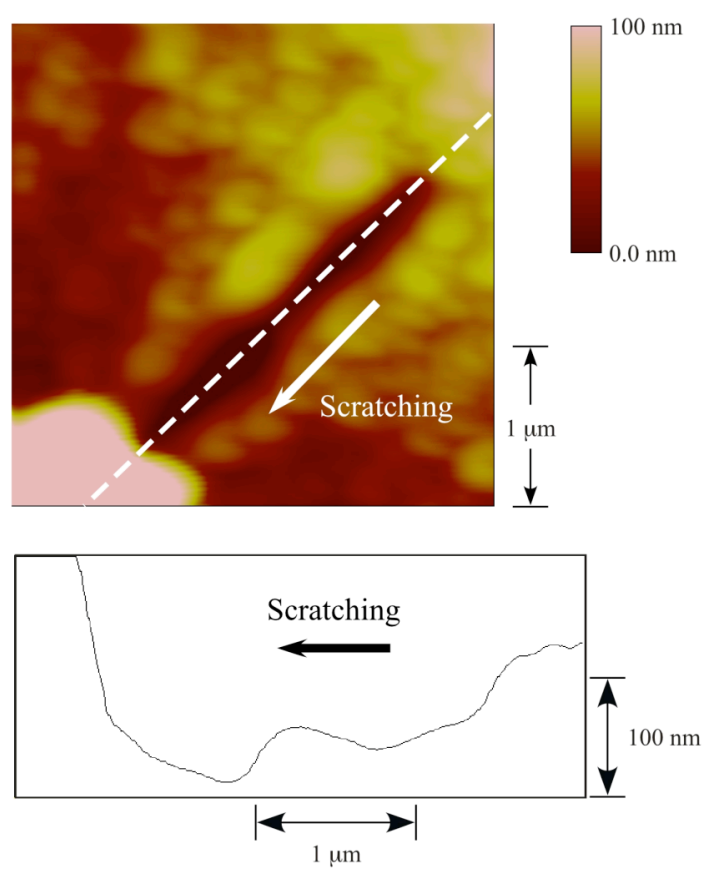

(b) Nano-scratching in air
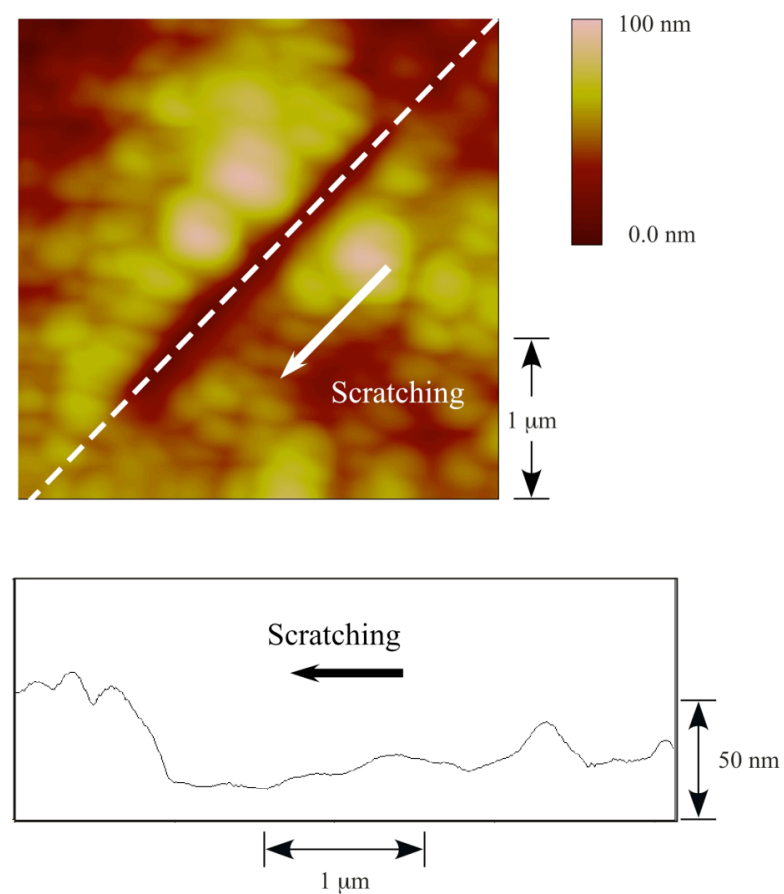

Figure 3. AFM images of the scratched areas after (a) nano-scratching in solution and (b) nano-scratching in air at a scratching rate of (a) $v=0.4 \mu \mathrm{m} \mathrm{s}^{-1}$ for the passive tantalum (100) surface subjected to anodic oxidation at $5.0 \mathrm{~V}$ (SHE) in $\mathrm{pH} 8.4$ borate buffer solution. The lower figures represent the cross sections of the grooves in the scratching direction drawn with the dashed lines in the AFM images. 
(a) Nano-scratching in solution

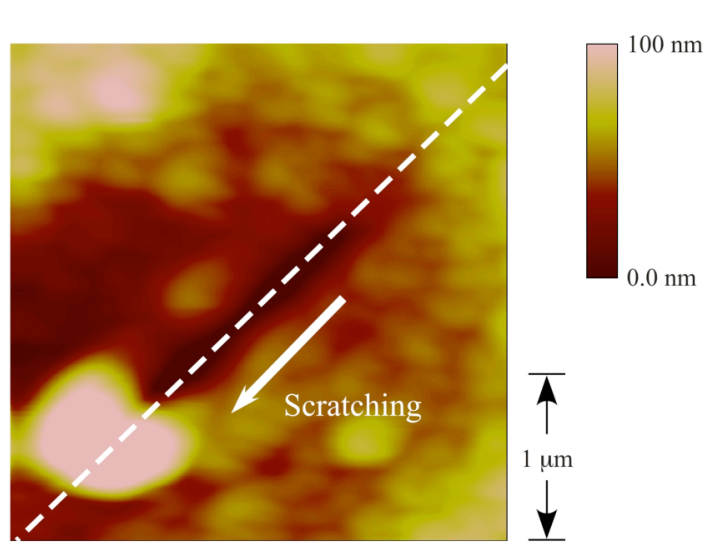

(b) Nano-indentation in air
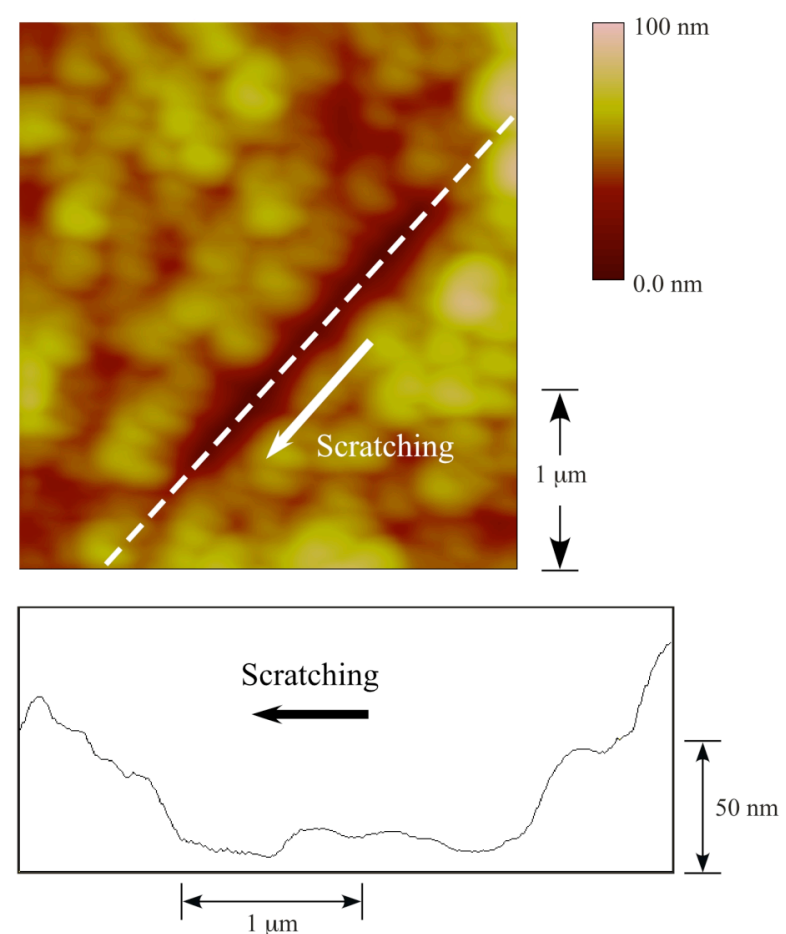

Figure 4. AFM images of the scratched areas after (a) nano-scratching in solution and (b) nano-scratching in air at a scratching rate of $v=0.8 \mu \mathrm{m} \mathrm{s}^{-1}$ for the passive tantalum (100) surface subjected to anodic oxidation at $5.0 \mathrm{~V}$ (SHE) in $\mathrm{pH} 8.4$ borate buffer solution. The lower figures represent the cross sections of the grooves in the scratching direction drawn with the dashed linse in the AFM images. 


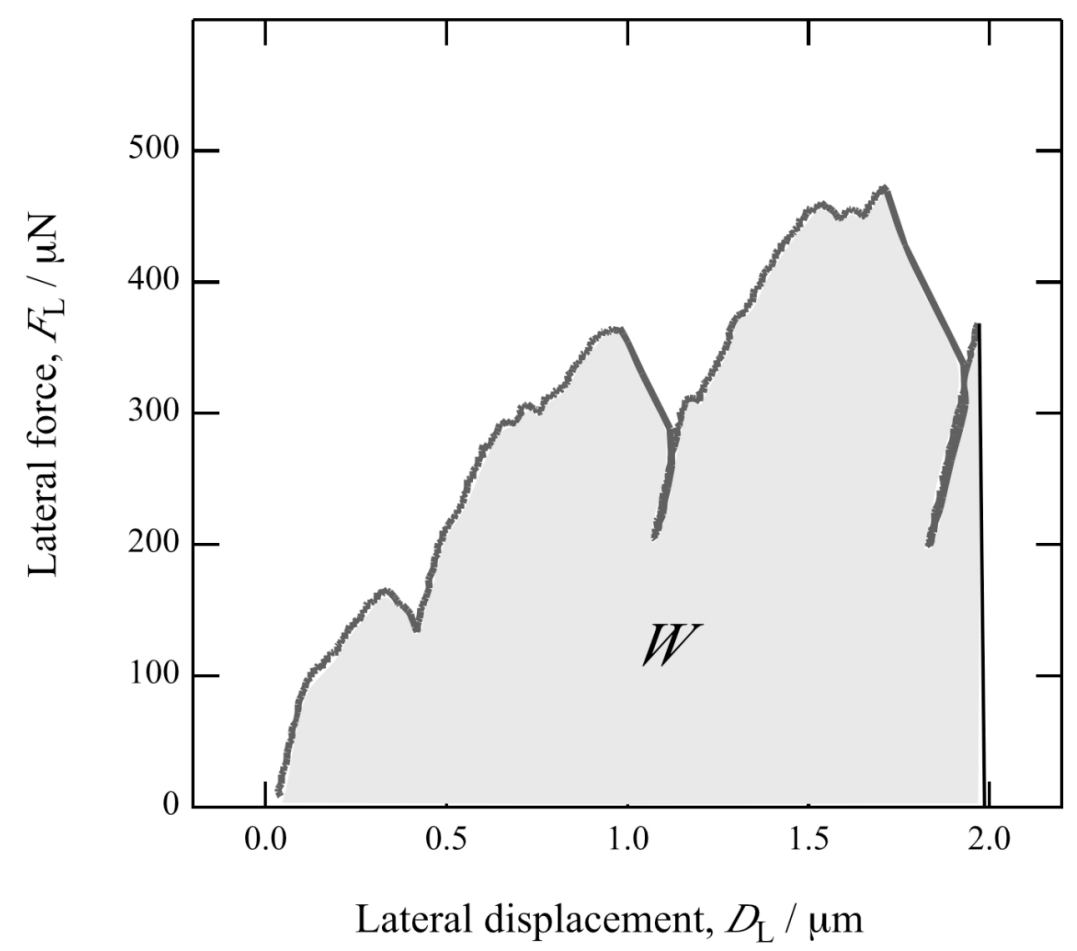

Figure 5. Irreversible work, $W$, done by the indeter tip during nano-scratching which is estimated by a graphic integration of the $F_{\mathrm{L}}$ vs. $D_{\mathrm{L}}$ curve from $D_{\mathrm{L}}=0$ to $2 \mu \mathrm{m}$. 


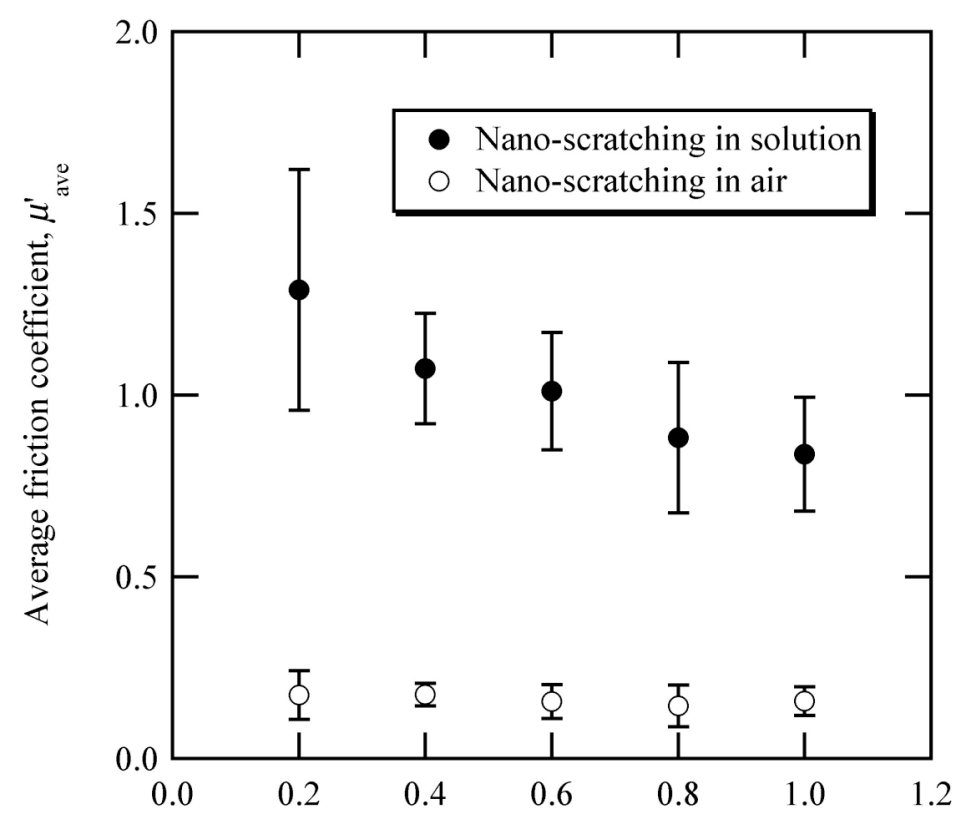

Scratching rate, $v / \mu \mathrm{m} \mathrm{s}^{-1}$

Figure 6. Relation between average friction coefficient, $\mu_{\text {ave }}^{\prime}$, and scratching rate, $v$, obtained with nano-scratching in solution and in air. 


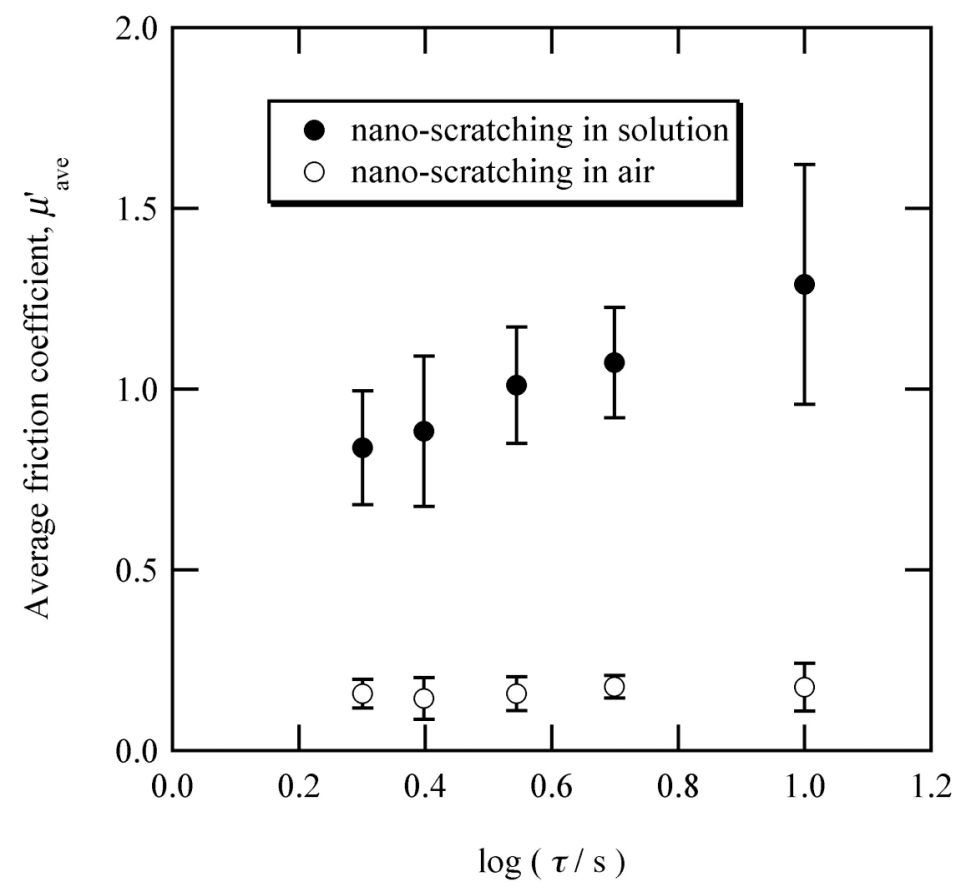

Figure 7. Relation between average friction coefficient, $\mu_{\text {ave }}^{\prime}$, and logarithm of time, $\tau$, required for scratching. 